\title{
Acute stent malfunction in a calcified lesion
}

\author{
Santosh Kumar Sinha MBBS MD DM, Ramesh Thakur DM, Mukesh Jitendra Jha MD, Asmutosh Kumar MD, \\ Vikash Mishra MD, Amit Goel MD, Chandra Mohan Varma DM, Vinay Krishna MCh
}

\begin{abstract}
SK Sinha, R Thakur, MJ Jha, et al. Acute stent malfunction in a calcified lesion. Curr Res Cardiol 2015;2(4):197-198.

Stent fracture is an emerging complication of coronary stenting. Stent fractures were originally reported in the superficial femoral arteries but have since been reported in almost all vascular sites. They are the result of the complex interplay among stent design, the stented segment, plaque morphology, deployment technique, including forceful exaggerated motion in the atrioventricular groove as observed in right coronary artery, long stent, tortuous lesion, stent overexpansion, complex lesion after stenting of a totally occluded vessel, and calcified lesions. Their presentation is highly
\end{abstract}

$\Delta$ fter bare metal stents, drug-eluting stents (DES) have emerged as $\mathrm{A}_{\text {principle devices for percutaneous coronary intervention. New }}$ concerns have emerged, including late stent thrombosis and stent fracture. These complications range from being asymptomatic in nature, detected on routine screening without any sequelae, to sudden cardiac death related to acute stent thrombosis. The true incidence of these two complications remains unknown because of limited sensitivity of angiography in detecting fracture. With the advent of stent boost, intravascular ultrasound, multidetector computed tomography, optical coherence tomography and cardiac magnetic resonance imaging, the incidence appears to be on the rise. Initially, these DESrelated complications were reported in superficial femoral arteries, but have since been reported in the coronary arteries as well.

\section{CASE PRESENTATION}

A diabetic, hypertensive 60-year-old woman presented with angina on exertion (chronic stable angina, Canadian Cardiovascular Society grade III) despite guideline-directed medical therapy. Her treadmill test was strongly positive for stress-induced myocardial ischemia. An electrocardiogram showed mild ST-T changes. Echocardiography revealed normal left ventricular systolic function with an ejection fraction of $60 \%$ and trivial mitral regurgitation. Her coronary angiogram was performed after informed consent, revealing critical calcified lesions of mid and distal left anterior descending arteries (LAD) (Figure 1). Percutaneous coronary intervention was subsequently planned. Intravenous heparin $(100 \mathrm{U} / \mathrm{kg})$ was administered. The left main coronary artery was hooked with a 6 Fr EBU Launcher Coronary Guide Catheter (Medtronic, USA). The lesion was crossed by a 0.014 inch $190 \mathrm{~cm} \mathrm{Hi-Torque} \mathrm{Balance} \mathrm{Middleweight} \mathrm{Elite} \mathrm{guide} \mathrm{wire}$ (Abbott Vascular, USA) with some difficulty because of the calcified lesion. The lesion was gradually predilated with $1.5 \mathrm{~mm} \times 10 \mathrm{~mm}$, $2 \mathrm{~mm} \times 10 \mathrm{~mm}$ and $2.5 \mathrm{~mm} \times 10 \mathrm{~mm}$ sprinter Legend RX balloons (Medtronic, USA). An Endeavour Resolute stent (zotarolimus-eluting stent; $2.5 \mathrm{~mm} \times 18 \mathrm{~mm}$; Medtronic, USA) was deployed at $10 \mathrm{~atm}$ distally. The proximal lesion was stented using an Endeavour Resolute stent at 12 atm (Figure 2). Subsequent angiogram revealed a grade $\mathrm{V}$ stent fracture of the proximal LAD stent with filling defect, ie, total separation (Figure 3), which was also confirmed on stent boost (Stent Boost Subtract, Philips Healthcare, The Netherlands) (Figures 4A and 4B). Calcium was also visible in stent boost (Figures 4A and 4B; white arrows). The stent was postdilated by a $2.75 \mathrm{~mm} \times 10 \mathrm{~mm}$ NC Track variable, ranging from asymptomatic, perforation, restenosis, migration of the stent to sudden cardiac death related to stent thrombosis. They have been reported as acute consequences as well as delayed presentation. Stent fractures were initially reported with bare metal stents but are now seen with drug-eluting stents as well. Herein, a case of stent fracture of a third-generation zotarolimus-eluting stent in a 60 -year-old woman is reported, with a moderately calcified lesion that was diagnosed by stent boost and successfully treated with restenting using another drug-eluting stent.

Key Words: Calcified lesion; Perforation; Restenosis; Stent fracture, Stent migration; Sudden cardiac death

balloon (Abbott, USA) at high pressure (up to $22 \mathrm{~atm}$ ). When it appeared to be adequately dilated, another Endeavour Resolute stent was deployed at $12 \mathrm{~atm}$ (Figures $4 \mathrm{C}$ and 5) and postdilated using a $2.75 \mathrm{~mm} \times 10 \mathrm{~mm} \mathrm{NC}$ Track balloon to 28 atm (Figures 4D and 6). The final angiogram revealed well-opposed stents with thrombolysis in myocardial infarction grade III flow, which was also confirmed by stent boost (Figures 4D and 7). She was discharged home after an uneventful hospital course and has been in regular follow-up since then.

\section{DISCUSSION}

Current registries have published the incidence of DES fracture ranging from $1.7 \%$ to $2.6 \%(1,2)$. Usually, stent fracture occurs in long stents implanted with a larger balloon at high pressure or at the site of overlapping stents, especially when placed in tortuous vessels or calcified lesions (3). However, fractures are also associated with stent design because they occur more often with long sirolimus-eluting stents compared with paclitaxel-eluting stents (2.9\% versus $0.5 \%)(2)$. Fractures are the outcome of the interplay among stent design, the stented segment, pulsatile and nonpulsatile biomechanical forces, and plaque morphology at a particular vascular site, stent length, vein graft, or a right coronary artery (RCA) lesion, because stents deployed in lesions located in the RCA are at an eight-fold increased risk for fracture compared with non-RCA stents (4). Stent fracture has been reported to be as high as $29 \%$ of the stented lesions at autopsy, which is higher than clinically reported. Stent fracture is classified as grade I (single-strut fracture), II ( $\geq 2$ struts), III ( $\geq 2$ struts with deformation), IV (with transection without gap) and V (with transection causing gap in stent segment) (5). Not all stent fractures are associated with clinical sequelae; on occasion, tissue overgrowth inside the stent may mask stent fracture making it 'asymptomatic', and may present as an incidental finding $(3,5)$. Symptomatic stent fractures can have a myriad of presentations such as clinical restenosis, stent thrombosis, recurrent angina, myocardial infarction and even sudden death (6-8). Maldistribution of the drug due to stent architecture malfunction following stent fracture is implicated in restenosis. Heavily calcified lesions often resist expansion, so the bed remains underprepared. If the lesion is diffuse, which may require a longer stent, then proper expansion also becomes difficult. Postdilation with a noncompliant balloon, as in our case, may cause a fracture to occur. Our patient's proximal and distal stent segments were completely separated with a distinct gap; therefore, this was an extremely uncommon type V stent fracture.

Department of Cardiology, LPS Institute of Cardiology, GSVM Medical College, Kampur, Uttar Pradesh, India

Correspondence: Dr Santosh K Sinha, Department of Cardiology, LPS Institute of Cardiology, GSVM Medical College, Kanpur, Uttar Pradesh 208002 India. Telephone 91-9670220088, e-mail fionasan@rediffmail.com 


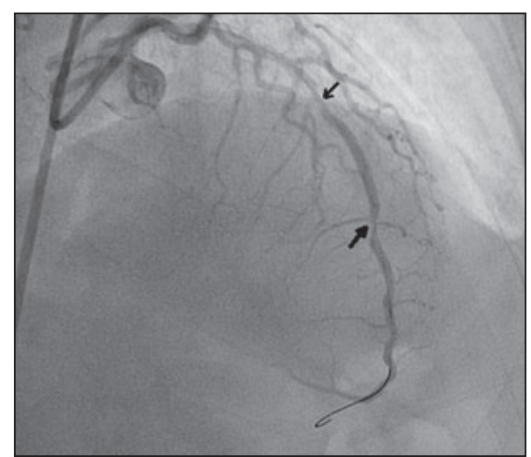

Figure 1) Critical calcified lesion of mid and distal left anterior descending artery

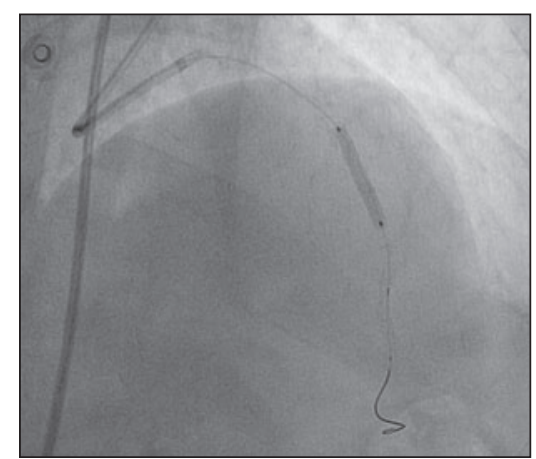

Figure 2) Proximal lesion being stented by an Endeavour Resolute stent

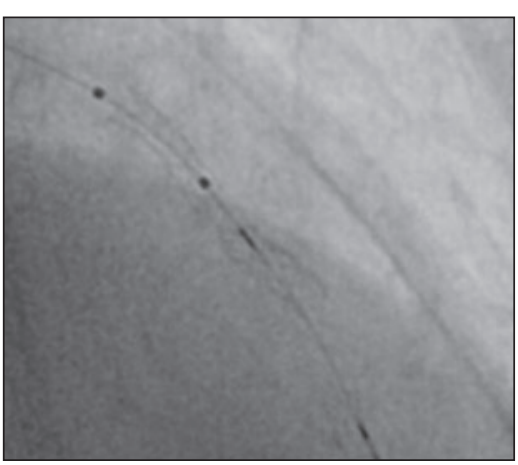

Figure 3) Grade $V$ stent fracture, ie, total separation of proximal stent on fluroscope

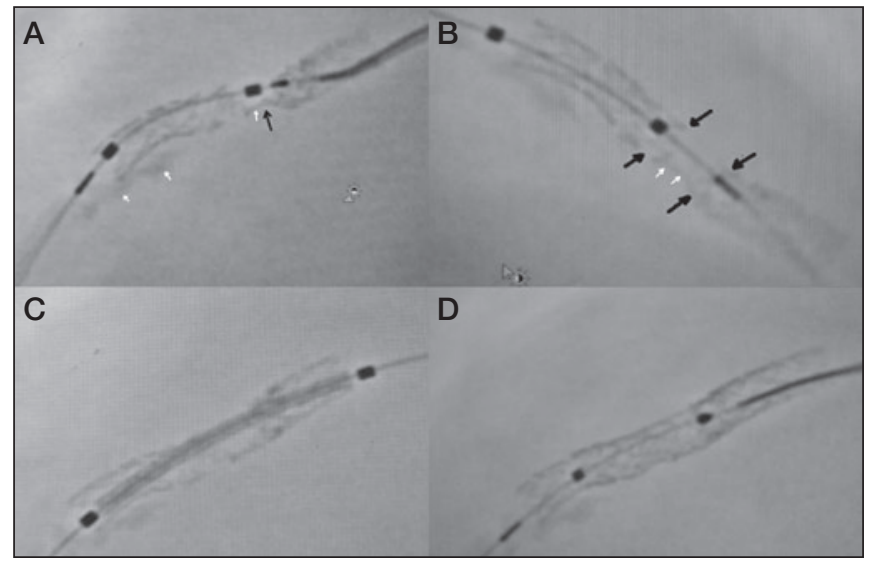

Figure 4) StentBoost images showing grade $V$ stent fracture (A and B); another stent positioned within fractured stent $(\mathrm{C})$; postdilation of new stent using noncompliant balloon. Final angiogram revealed well-opposed stents with thrombolysis in myocardial infarction III flow (D). White arrows indicate calcium
Overdilation and overstretching may also have been contributory. It is interesting to note that even third-generation stents are not immune to fracture; factors behind stent fracture also apply. Furthermore, stent fractures occur in the LAD as well. In type V fracture, reintervention is needed because of the high potentional for serious sequelae. Although different diagnostic modalities currently exist, stent visibility is limited on conventional fluoroscopic imaging. A patient's physique, a stent's platform and stent thickness also contribute to radiopacity. StentBoost technology enables improved visibility of stent struts. StentBoost images are chiefly used to evaluate the expansion of a stent after deployment $(9,10)$. The technology can also be used to position a stent precisely over a previously stented segment, as in our patient. The need for StentBoost technology also arises when a DES needs to be deployed because of in-stent restenosis of a bare metal stent. The identification of stent fractures is an emerging application of the StentBoost imaging system.

DISCLOSURES: The authors have no financial disclosures or conflicts of interest to declare.

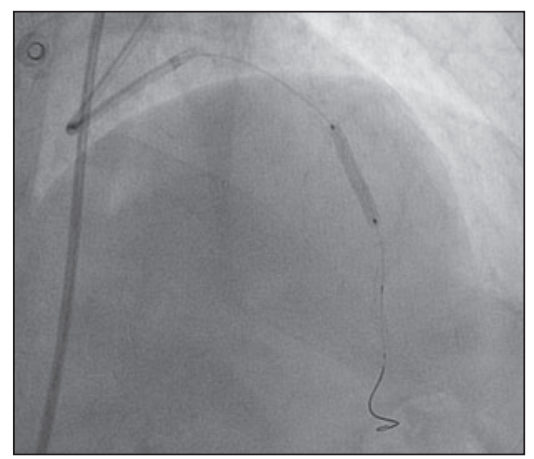

Figure 5) Another stent being deployed in proximal stent after its fracture

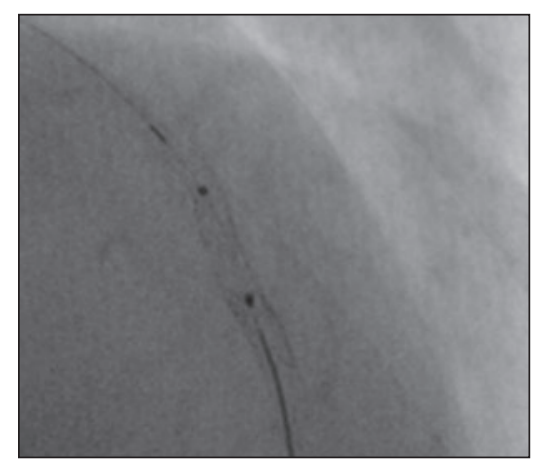

Figure 6) High-pressure postdilation of new stent using noncompliant

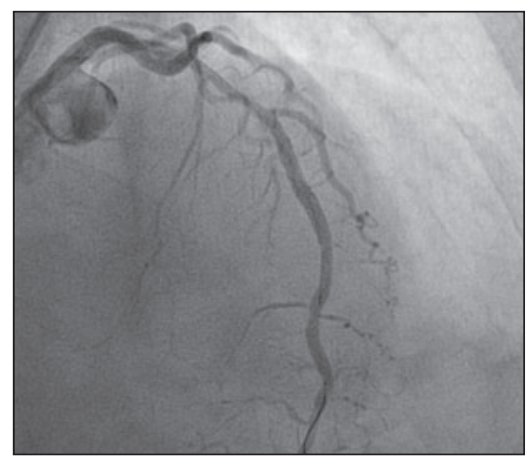

Figure 7) Final angiogram revealed well-opposed stents with thrombolysis in myocardial infarction III flow in left anterior descending artery

\section{REFERENCES}

1. Aoki J, Nakazawa G, Tanabe K, et al. Incidence and clinical impact of coronary stent fracture after sirolimus-eluting stent implantation. Catheter Cardiovasc Interv 2007:69:380-6.

2. Lee MS, Jurewitz D, Aragon J, Forrester J, Makkar RR, Kar S. Stent fracture associated with drug-eluting stents: Clinical characteristics and implications. Catheter Cardiovasc Interv 2007;69:387-94.

3. Sianos G, Hofma S, Ligthart JM, et al. Stent fracture and restenosis in the drugeluting stent era. Catheter Cardiovasc Interv 2004;61:111-6.

4. Kim HS, Kim YH, Lee SW, et al. Incidence and predictors of drug-eluting stent fractures in long coronary disease. Int J Cardiol 2009;133:354-8.

5. Nakazawa G, Finn AV, Vorpahl M, et al. Incidence and predictors of drug-eluting stent fracture in human coronary artery a pathologic analysis. J Am Coll Cardiol 2009;54:1924-31.
6. Shite J, Matsumoto D, Yokoyama M. Sirolimus-eluting stent fracture with thrombus, visualization by optical coherence tomography. Eur Heart J 2006;27:1389.

7. Park J-S, Shin D-G, Kim Y-J, Hong G-R, Cho I-H. Acute myocardial infarction as a consequence of stent fracture and plaque rupture after sirolimus-eluting stent implantation. Int J Cardiol 2009;134:e79-81.

8. Makaryus AN, Lefkowitz L, Lee ADK. Coronary artery stent fracture. Int J Cardiovasc Imaging 2007;23:305-9.

9. Kang WY, Kim W, Hwang SH, Kim W. Dark side of drugeluting stent: Multiple stent fractures and sudden death. Int J Cardiol 2009;132:e125-7.

10. Shinde RS, Hardas S, Grant PK, Makhale CN, Shinde SN, Durairaj M. Stent fracture detected with a novel fluoroscopic stent visualization technique - StentBoost. Can J Cardiol 2009;25:487. 